\title{
RF-Energy Harvesting and Wireless Power Transfer Efficiency from Digitally Modulated Signals
}

\author{
Javier Blanco, Ferran Bolos, Ana Collado, Apostolos Georgiadis \\ Department of Microwave Systems and Nanotechnology \\ Centre Tecnologic de Telecomunicacions de Catalunya (CTTC) \\ 08860 Castelldefels, Barcelona, Spain \\ ageorgiadis@cttc.es
}

\begin{abstract}
The RF-DC conversion efficiency of an RF harvesting circuit when it is excited by digitally modulated signals is investigated. A variety of signals with the same average power but different complementary cumulative distribution function (CCDF) and peak-to-average-power ratio (PAPR) properties are applied at the input of a prototype UHF diode rectifier. The rectifier is optimized for maximum efficiency under low average input power levels and the RF-DC conversion efficiency for different output load conditions is evaluated. It is shown that depending on the CCDF and PAPR properties of the applied signal a different optimum load exists which leads to a maximum efficiency.
\end{abstract}

Keywords-energy harvesting; wireless power transfer; rectifier; complementary cumulative distribution function (CCDF); Peak-to-average-power ratio (PAPR); digital modulation

\section{INTRODUCTION}

A lot of recent research works have focused on investigating the RF-DC conversion efficiency of rectifier circuits due to the increased number of applications which are related to the Internet of Things, RFID or wireless power transmission technologies (WPT) [1][2][3]. Following the paradigm of RFID technology, several new types of wireless systems are being developed such as WiCoPT [4] or SWIPT [5][6], that transmit power and information simultaneously using modulated signals. In this work, we study the RF-DC conversion efficiency of a rectifier using modulated signals, making a first step to maximize the power transfer efficiency based on the used signal properties and output load.

Recent works have already shown that the RF-DC conversion efficiency of a rectifier is strongly affected by the underlying signal PAPR [7][8][9]. In this work, the effect of the CCDF distribution as well as its PAPR on the optimum load is investigated. In addition, it is shown that as the baseband filter roll-off factor $(\beta)$ affects the signal PAPR, it also provides a means to control the RF-DC conversion efficiency of a WPT system.

The paper is structured as follows: in section II the rectifier prototype is described. In section III, the input signal parameters and characteristics are defined and discussed. In section IV the measurement results are presented, and finally, in section $\mathrm{V}$ the conclusions of this work are summarized.

\section{RECTIFIER DESIGN}

A UHF rectifier was designed and optimized using harmonic balance simulation. The circuit, shown in Fig. 1, consists of an RF source with $R_{s}=50 \Omega$, connected in series to a Schottky diode (D) followed by a shunt capacitor $\left(\mathrm{C}_{\mathrm{L}}\right)$ and a resistive load $\left(\mathrm{R}_{\mathrm{L}}\right)$. A matching network and harmonic filter was placed between the source and the diode in order to maximize power transfer, filter undesired harmonics and maximize RF-DC conversion efficiency. The rectifier efficiency is defined as the ratio between the dc power delivered to the load $\mathrm{P}_{\mathrm{L}, \mathrm{dc}}$ over the average RF power delivered by the source $\mathrm{P}_{\mathrm{A}}$ :

$$
\eta=\frac{P_{L, d c}}{P_{A}}=\frac{V_{L}^{2}}{R_{L} P_{A}}
$$

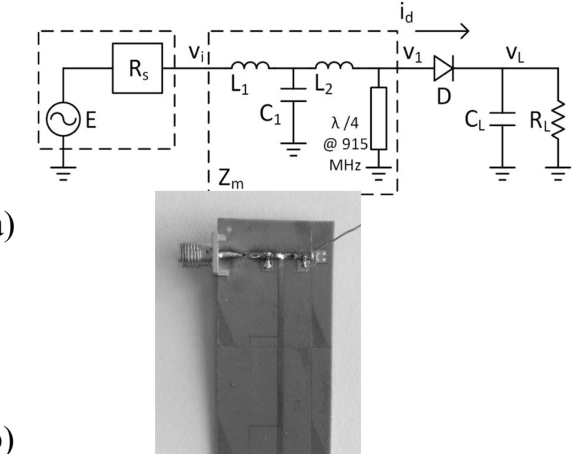

Fig. 1. Designed rectifier: a) circuit diagram, b) fabricated prototype.

The rectifier was based on the Skyworks SMS7630 Schottky diode. A T-type matching network was used consisting of two off-the-shelf series inductors and one shunt capacitor. The inductors were $\mathrm{L1}=3.3 \mathrm{nH}$ (Coilcraft 04CS3N3) and $\mathrm{L} 2=43 \mathrm{nH}$ (Coilcraft 06CS430) and the capacitor $\mathrm{Cl}=3 \mathrm{pF}$ (Murata GMR39C0G030C050). The values of the capacitor and the two inductors were optimized simultaneously with the output load in order to maximize the efficiency for a fixed average available signal power $\mathrm{P}_{\mathrm{A}}=-20$ $\mathrm{dBm}$. The harmonic filter consisted of a shorted stub a quarter wavelength long at the fundamental frequency of $915 \mathrm{MHz}$. It provides a short at dc and at the second harmonic frequency. At the output of the rectifier a $10 \mathrm{nF}$ (Murata) shunt capacitor was placed, and a $20 \mathrm{~K} \Omega$ trimmer (variable) resistor is used as the rectifier load. The combination of both elements is a low pass filter which only allows the dc component of the signal. The circuit was built on a 20 mil thick Arlon A25N substrate with 
dielectric permittivity of 3.38 and loss tangent of 0.0025 . In Fig. $1 \mathrm{~b}$ a photo of the fabricated prototype is shown.

\section{SIGNAL DESCRIPTION}

The complementary cumulative distribution function (CCDF) expresses the probability that the instantaneous power of a signal has a higher or equal value than the CCDF argument. The CCDF argument is given as the ratio of instantaneous power over average power. The signal peak to average power ratio (PAPR) can be identified from its CCDF curve. In this paper we considered as PAPR the argument of CCDF corresponding to a $0.01 \%$ probability. In order to evaluate the CCDF of several modulated signals we have setup a test bench shown in (Fig. 2). An Agilent E4438C function generator is used as a transmitter, whereas the receiver consists of an Agilent E4448AVSA spectrum analyzer linked to Vector Signal Analyzer software (VSA).

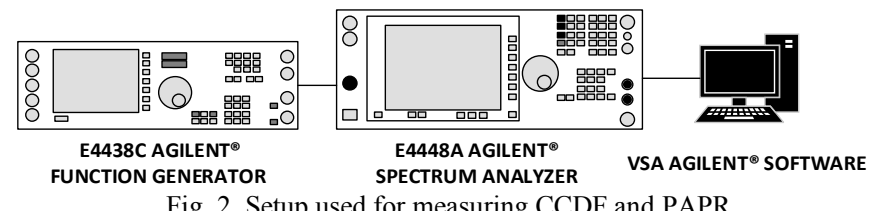

The baseband shaping filter used in the transmitter is a root raised cosine filter with roll-off factor $\beta$. The measured CCDF of different modulated signals is shown in Fig. 3, where the different shape of the CCDF curve corresponding to different modulation formats can be appreciated for $\beta=0$ and a symbol rate of $500 \mathrm{KBPS}$. BPSK and 64QAM show larger CCDF values for the same $\mathrm{P} / \mathrm{P}_{\mathrm{A}}$ compared to $8 \mathrm{PSK}$ and QPSK.

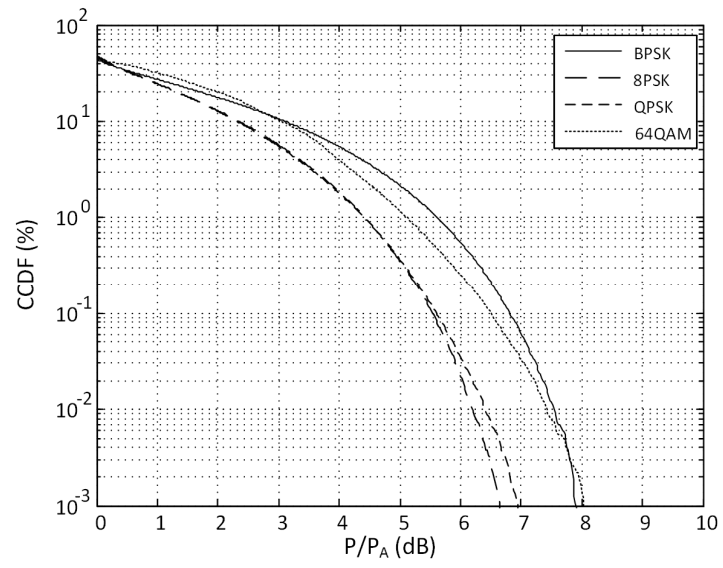

Fig. 3. Measured CCDF of modulated signals with $\beta=0$ and $500 \mathrm{KBPS}$ symbol rate.

The PAPR of the transmitted signal decreases as the value of $\beta$ increases from a value of 0 up to approximately 0.4 , while it varies very little for $\beta$ values in the 0.4-1.0 range, as shown in Fig. 4. For instance, considering BPSK modulation a $3.7 \mathrm{~dB}$ reduction in PAPR is observed in the PAPR when $\beta$ increases from 0 to 0.35 . Therefore, the roll-off factor has a strong effect on PAPR, which will influence the RF-DC conversion efficiency as will be shown in section IV. The symbol rate of the signal however, does not have any appreciable effect on the CCDF curve and the PAPR as shown in Fig. 5.

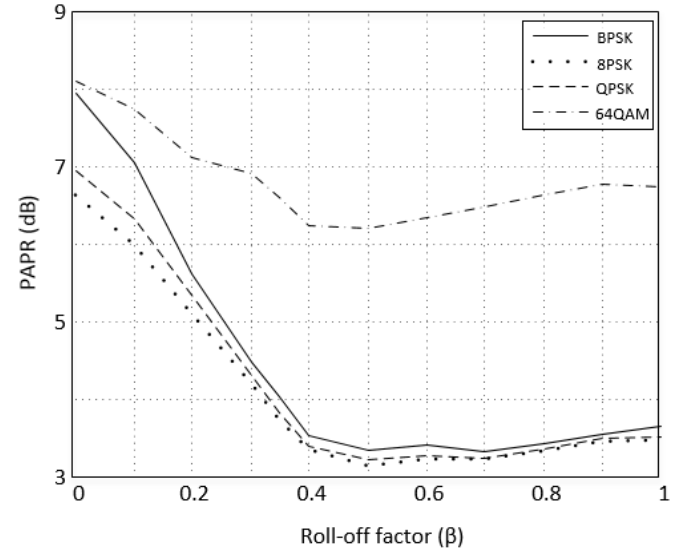

Fig. 4. Measured PAPR for different roll-off factors $\beta$ (500 KBPS).

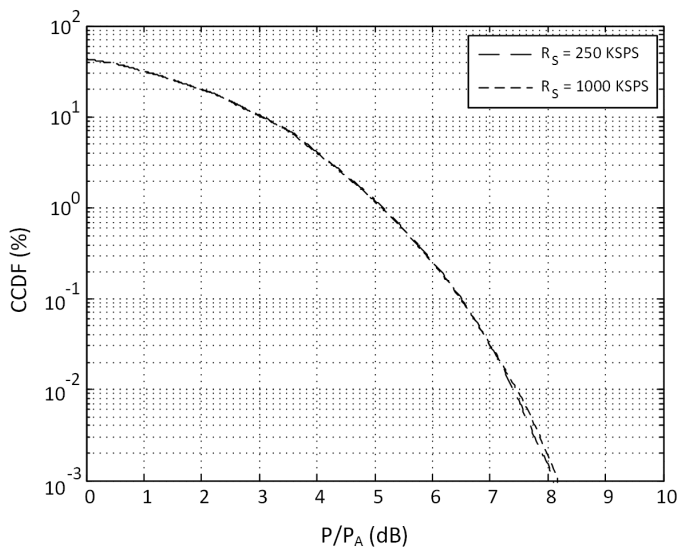

Fig. 5. Measured CCDF comparing 64QAM modulated signals with different symbol rate.

It is therefore possible to control the shape of the CCDF curve and the PAPR up to certain level, by selecting the baseband filter roll-off factor. Fig. 6 compares the measured CCDF curves of a 64QAM modulated signal of $500 \mathrm{KSPS}$ with $\beta=0.5$ and $\beta=1.0$. Fig. 7 compares the CCDF of a 64QAM with roll-off factor 0.5 and a 8 PSK signal with roll-factor of 1.0. The two waveforms lead to the same PAPR but different CCDF shapes and instantaneous power variance.

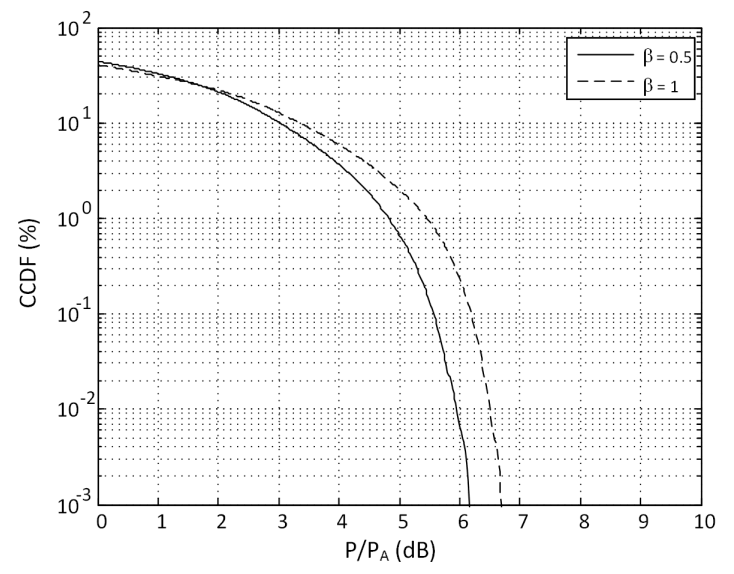

Fig. 6. Measured CCDF comparing 64QAM with different $\beta$ (500 KBPS). 


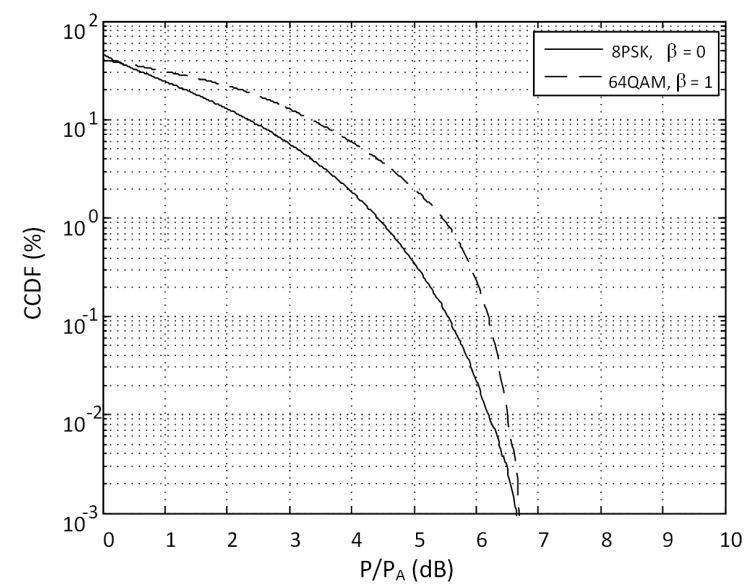

Fig. 7. Measured CCDF comparing 64QAM with $\beta=1$ and 8PSK with $\beta=0$ (500 KBPS).

\section{MEASUREMENTS}

In order to measure the efficiency of the rectifier, a second test bench was set-up, shown in Fig. 8. In this case the receiver is the rectifier circuit, and a PROMAX MD-200B digital multimeter is used to measure the rectifier output dc voltage. Subsequently the RF-DC conversion efficiency is computed using (1).

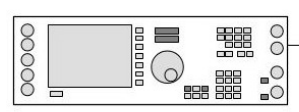

E4438C AGILENT ${ }^{\circledast}$ FUNCTION GENERATOR

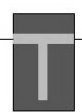

RECTIFIER

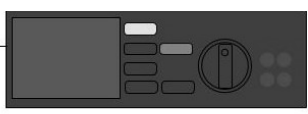

MD-200B AGILENT ${ }^{\circledR}$ DIGITAL MULTIMETER
Fig. 8. Set-up used for measuring the rectifer RF-DC conversion efficiency.

The rectifier RF-DC conversion efficiency obtained for different modulated signals of the same average power versus different output load $\mathrm{R}_{\mathrm{L}}$ values is shown in Fig. 9. The performance of the various modulated signals is also compared to that of a continuous wave (CW) input signal. One can see that the load value corresponding to a maximum efficiency is different for each of the applied signals. There is a region corresponding to lower $\mathrm{R}_{\mathrm{L}}$ values where $\mathrm{CW}$ has better RF-DC conversion efficiency than modulated signals with higher PAPR. As the load is increased, the efficiency of the $\mathrm{CW}$ signal evenually begins to decrease and the efficiency of modulated signals becomes better than the CW. Specifically, signals with higher PAPR present better efficiency at higher load values. As an example, with a $1 \mathrm{~K} \Omega$ load the efficiency of a 64QAM signal is $1 \%$ worse than that of a $\mathrm{CW}$ signal, but if the load is increased to $15 \mathrm{~K} \Omega$ the efficiency obtained with a 64QAM signal is $3 \%$ better than the $\mathrm{CW}$ signal.

The same trend was observed at different input (average) power levels of $-20 \mathrm{dBm}$ and $0 \mathrm{dBm}$. As the average power is increased the RF-DC efficiency values are larger for all signals considered. If the power is increased beyond $0 \mathrm{dBm}$ the efficiency begins to drop due to the breakdown voltage of the diode. Furthermore, as the average input power is increased the load value $R_{L}$ corresponding to a maximum efficiency is reduced for all considered signals.

In Fig. 10 the RF-DC conversion efficiency of 64 QAM signals with $\beta=0.5$ and $\beta=1.0$ is compared for varying load values $R_{L}$. The CCDF of these two signals was presented in Fig. 6 where it was shown that using $\beta=1.0$ resulted in larger CCDF values and slightly higher PAPR. Similarly to the results of Fig. 9, the signal with lower PAPR has a smaller optimum load than the signal with the higher PAPR and consequently it has a larger RF-DC conversion efficiency at smaller load $R_{L}$ values while the signal with the higher PAPR has better efficiency at larger $\mathrm{R}_{\mathrm{L}}$.

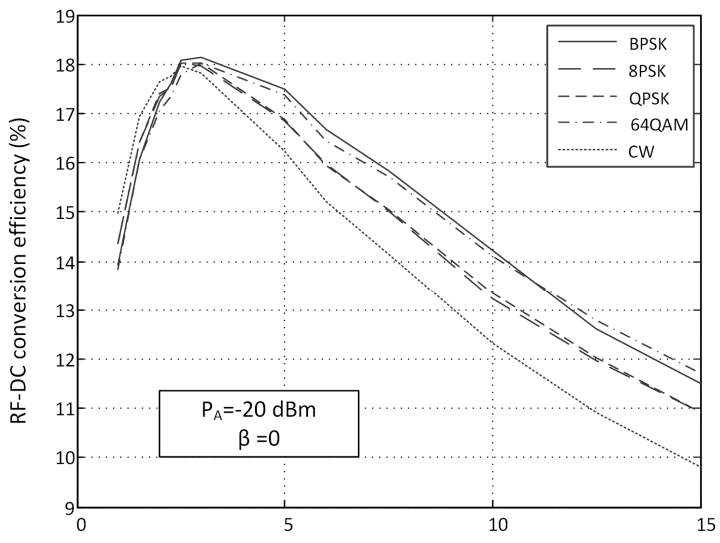

a)

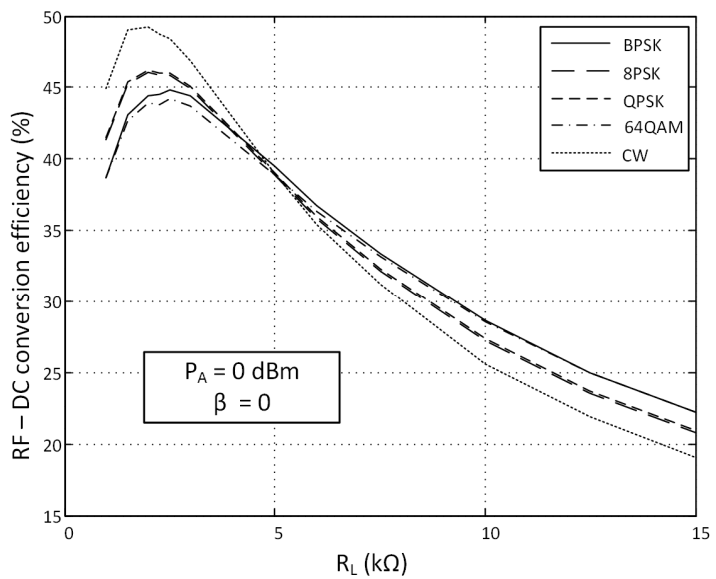

b)

Fig. 9. Measured RF-DC conversion efficiency of randomly digital modulated signals with symbol rate 500 KSPS versus $R_{L}$. a) $P_{A}=-20 \mathrm{dBm}$, b) $P_{A}=0$ $\mathrm{dBm}$.

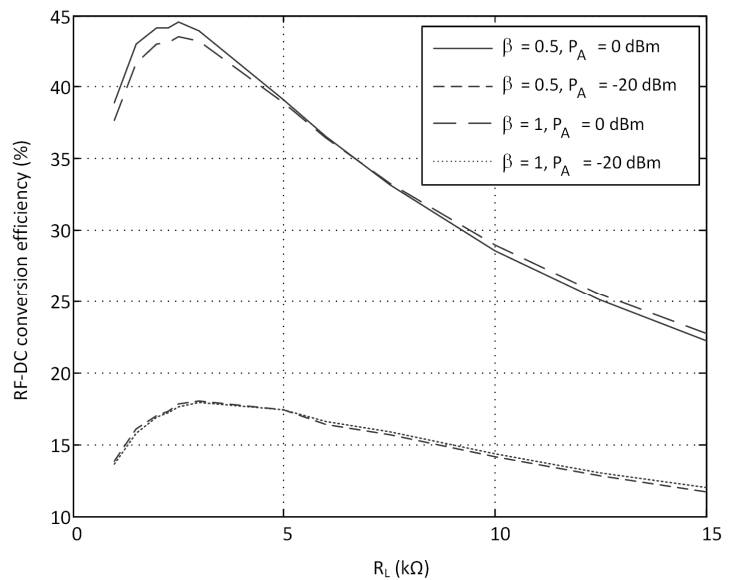

Fig. 10. Measured RF-DC conversion efficiency of 64QAM with different $\beta$.

In Fig. 11 we compare a 64QAM signal with $\beta=1$ and a 8PSK signal with $\beta=0$, which, as shown in Fig. 7 , have the 
same PAPR but different CCDF curves. Specifically, the 64QAM signal has larger CCDF values but its 'waterfall' curve drops suddenly as the peak power values are increased and results to the same PAPR as the 8PSK signal which has a smoother CCDF variation. In this case, it is observed that the 64 QAM signal leads to a higher RF-DC conversion efficiency at larger load $\mathrm{R}_{\mathrm{L}}$ values.

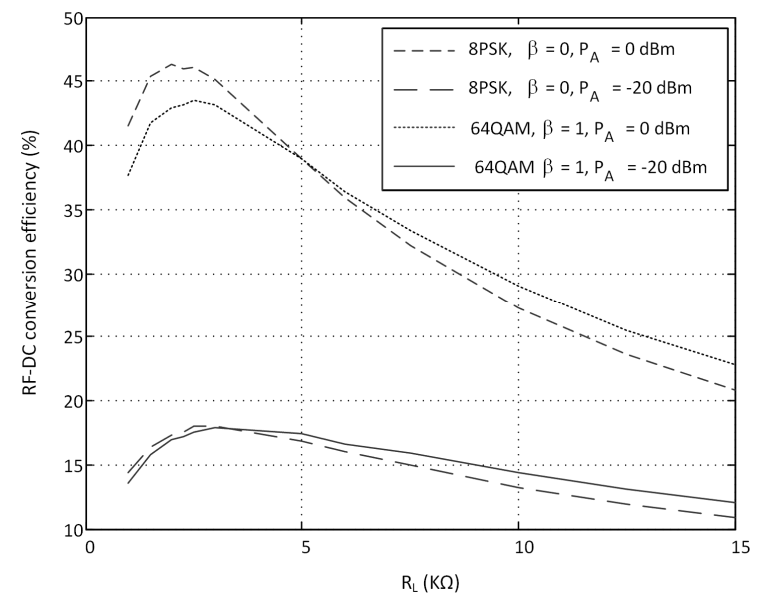

Fig. 11. Measured RF-DC conversion efficiency of 64QAM with $\beta=1$ and 8PSK with $\beta=0$.

Finally, Fig. 12 shows the RF-DC conversion efficiency of a 64QAM signal versus the symbol rate for three different load values. It was shown in Section III that the modulation rate does not affect its CCDF curve and PAPR value. In Fig. 12 however, it is observed that the RF-DC efficiency initially increases with increasing symbol rate while it appears to reach a maximum value for a certain symbol rate. This is attributed to the effect of the low pass filter at the output of the rectifier. If the symbol rate increases further a reduction in the RF-DC conversion efficiency is expected due to the limited bandwidth of the rectifier matching network.

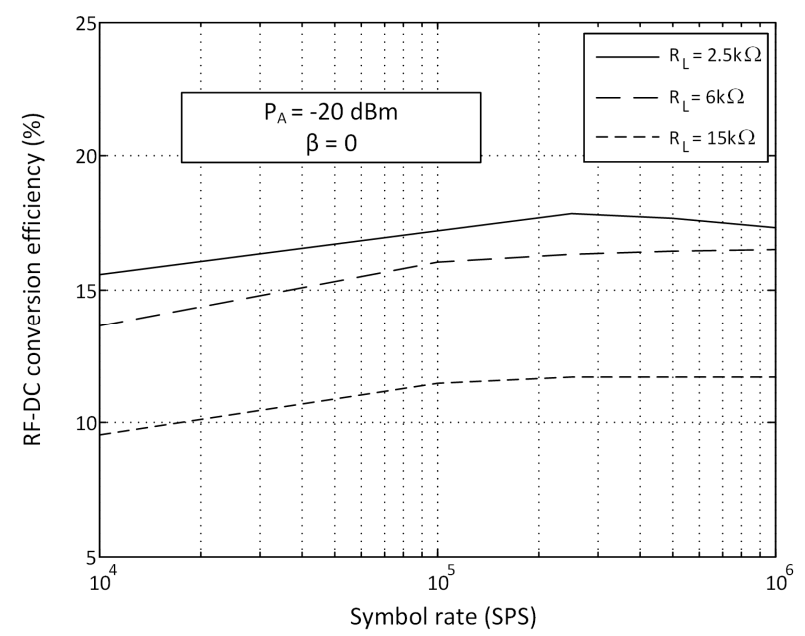

Fig. 12. Measured RF-DC conversion efficiency of 64QAM versus Symbol rate for different $R_{L}$.

\section{CONCLUSIONS}

The effect of randomly modulated signals on the RF-DC conversion efficiency of a UHF rectifier circuit was studied. It was observed that the optimum load corresponding to a maximum RF-DC conversion efficiency increases with increasing PAPR of the input signal. As a result, signals with lower PAPR tend to lead to higher efficiency at lower load values while for large load values the efficiency obtained with signals having a high PAPR is better. It is also observed that while PAPR is a good indicator of the RF-DC efficiency performance, the complete CCDF curve, or other statistical measures such as the instantaneous power variance is necessary for an accurate evaluation of its behavior. Finally, the RF-DC conversion efficiency depends on the modulation rate through the output filter of the rectifier formed by the output capacitor and load value.

\section{ACKNOWLEDGMENT}

This work was supported by GenCat grant 2014 SGR 1551 and by the Spanish MEC and FEDER funds through project TEC2012-39143. The work was performed under the framework of EU COST Action IC1301 Wireless Power Transmission for Sustainable Electronics (WiPE).

\section{REFERENCES}

[1] J.A. Hagerty, F.B. Helmbrecht, W.H. McCalpin, R. Zane, Z.B. Popovic, "Recycling ambient microwave energy with broad-band rectenna arrays," IEEE Transactions on Microwave Theory and Techniques, vol. 52, no. 3, pp. 1014-1024, Mar. 2004.

[2] S. Kim, R. Vyas, J. Bito, K. Niotaki, A. Collado, A. Georgiadis, M.M. Tentzeris, "Ambient RF Energy-Harvesting Technologies for SelfSustainable Standalone Wireless Sensor Platforms," Proceedings of the IEEE, vol. 102, no. 11, pp. 1649-1666, Nov. 2014.

[3] M. Dini, M. Filippi, A. Costanzo, A. Romani, M. Tartagni, M. Del Prete, D. Masotti, "A fully-autonomous integrated rf energy harvesting system for wearable applications," in Proc. 2013 European Microwave Conference (EuMC),pp. 987-990, 6-10 Oct. 2013.

[4] S. Kawasaki, "The green energy harvesting winds by the RF/microwave power transmission," in Proc. 2013 IEEE Wireless Power Transfer Conference (WPTC), pp. 111-114, 15-16 May 2013.

[5] I. Krikidis, S. Timotheou, S. Nikolaou, G. Zheng; D.W.K. Ng, R. Schober, "Simultaneous wireless information and power transfer in modern communication systems," IEEE Communications Magazine, vol. 52, no. 11, pp. 104-110, Nov. 2014.

[6] B. Clerckx, E. Bayguzina, D. Yates, P.D. Mitcheson, "Waveform Optimization for Wireless Power Transfer with Nonlinear Energy Harvester Modeling", in Proc. 2015 IEEE International Symposium on Wireless Communication Systems (ISWCS), Brussels, Belgium, 25-28 Aug. 2015.

[7] M.S. Trotter, J.D. Griffin, G.D. Durgin, "Power-optimized waveforms for improving the range and reliability of RFID systems," in Proc 2009 IEEE International Conference on RFID, pp. 80-87, 27-28 April 2009.

[8] A. Collado, A. Georgiadis, "Optimal Waveforms for Efficient Wireless Power Transmission," IEEE Microwave and Wireless Components Letters,vol. 24, no. 5, pp. 354-356, May 2014.

[9] A. Boaventura, D. Belo, R. Fernandes, A. Collado, A. Georgiadis, N.B. Carvalho, "Boosting the Efficiency: Unconventional Waveform Design for Efficient Wireless Power Transfer," IEEE Microwave Magazine, vol. 16, no. 3, pp. 87-96, Apr. 2015. 